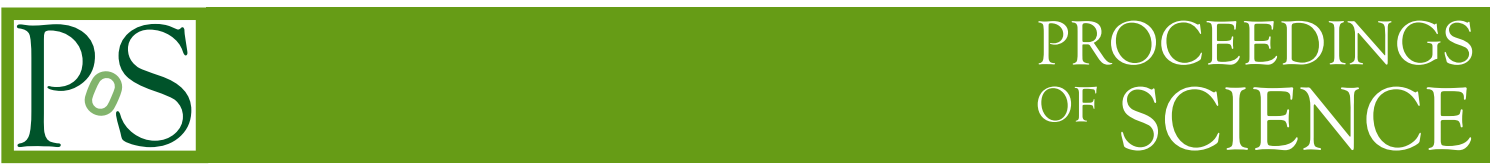

\title{
Extreme regimes of emission in relativistic outflows
}

\author{
E.V. Derishev* \\ Institute of Applied Physics RAS, 46 Ulyanov st., 603950 Nizhny Novgorod, Russia \\ E-mail: derishev@appl.sci-nnov.ru
}

\begin{abstract}
All broad-band blazar spectra display two broad and widely separated maxima. We analyse existing models, which attempt to explain non-thermal emission of blazars addressing the origin of these maxima. For various reasons that we discuss in this paper, none of these models can claim success with the entire blazar population and rather take responsibility for either $\mathrm{GeV}$ or $\mathrm{TeV}$ blazars. In addition, we analyse particle acceleration and radiation processes within joint framework, discuss extreme regimes of radiation and physical limits to the parameters of blazars' emitting zones, and show that in many cases requirements of blazar emission models cannot fit into these fundamental limits.

We also consider recent observation of sub-TeV emission from Gamma-Ray Burst afterglow in the case of GRB 190114C. Derived parameters for the emitting zone of this burst indicate that absorption of sub- $\mathrm{TeV}$ radiation inside the emitting zone is an important effect and are in agreement with predictions of the pair-balance model of relativistic shocks.

Based on this evidence, we argue that that relativistic flows (not only in GRBs, but in a general situation as well) are very efficient in accelerating electrons and that the energy of accelerated electrons is limited not by the acceleration process itself, but rather by the back reaction from the high-energy radiation produced in the flow.
\end{abstract}

High Energy Phenomena in Relativistic Outflows VII - HEPRO VII

9-12 July 2019

Facultat de Física, Universitat de Barcelona, Spain

* Speaker. 


\section{Introduction}

Blazars are one of the observational appearances of Active Galactic Nuclei (AGNs). Since the classical paper (Urry \& Padovani, 1995) it is widely accepted that AGNs' central engines consist of a super-massive black hole with an accretion disk around it. The matter falling from the disk to the black hole feeds two relativistic jets, escaping in opposite directions perpendicular to the plane of the disk. The jets (via one or another acceleration mechanism) produce high-energy particles, which then radiate and the radiation is beamed along the jet axis, being boosted in frequency and especially in brightness due to relativistic motion of the emitting zone connected to the jet material. When one is lucky enough to see an AGN with the jets aligned with the line of sight, then a blazar is observed.

All blazar spectra are exceptionally broad and clearly non-thermal, spanning from radio frequencies to $\mathrm{GeV}$ and sometimes $\mathrm{TeV}$ energies. The spectra are devoid of narrow features and display two broad maxima separated by $8-10$ orders in frequency. Observation-wise, the blazars form two populations with relatively few objects in between (see Fig. (1) from Finke (2013)): the so-called GeV blazars have low-frequency peak position clustered around around $1 \mathrm{eV}$ and highfrequency peak in sub-GeV - few $\mathrm{GeV}$ range, whereas the $\mathrm{TeV}$ blazars have low-frequency peak position clustered around around $1 \mathrm{keV}$ and high-frequency peak in sub- $\mathrm{TeV}$ region. $\mathrm{GeV}$ blazars are brighter on average, by almost 2 orders of magnitude, and their luminosity is dominated by the high-frequency peak. Luminosity of $\mathrm{TeV}$ blazars, on the contrary, is dominated by the lowfrequency peak.
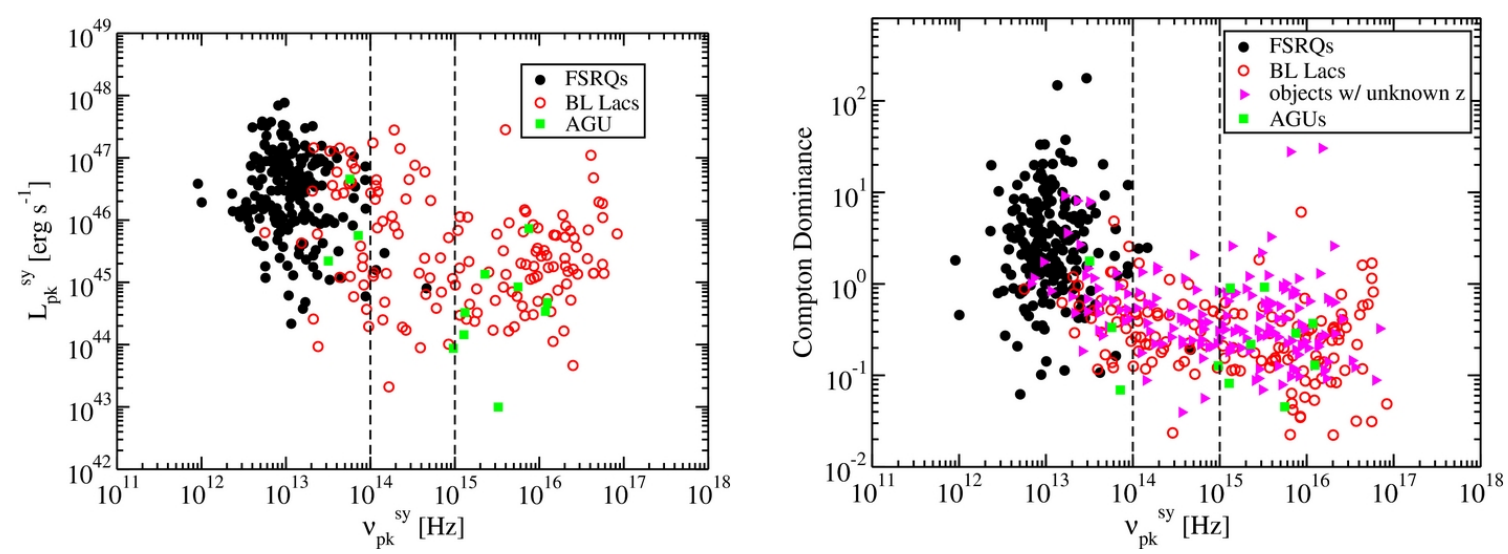

Figure 1: Left panel: peak synchrotron luminosity vs. peak synchrotron frequency. Right panel: Compton dominance (i.e., $L_{p k}^{C} / L_{p k}^{s y}$ ) vs. peak synchrotron frequency. Both figures are taken from Finke (2013).

There are three competing models, which attempt to explain blazar spectra (see, e.g., Abdo et al. (2010) for a review on blazar spectra). In the synchrotron-self-Compton (SSC) model (see, e.g., Ghisellini \& Maraschi (1989)) the low-frequency peak is attributed to synchrotron radiation from accelerated electrons; this synchrotron radiation is then upscattered by the same electrons to produce another, high-frequency peak, which is considered to be inverse Compton (IC) emission. The SSC model is usually being applied to TeV blazars; the values of emitting zone parameters required in this model for $\mathrm{GeV}$ blazars are hard to reconcile with observations (see below). The model with comptonization of external radiation (ERC; see, e.g., Sikora et al. (1994)) is similar 
to the SSC model, but it links the origin of high-frequency peak to comptonization of external radiation produced, for example, by the AGN's disk. The ERC model is usually applied to GeV blazars; their $\mathrm{TeV}$ counterparts apparently lack sufficiently luminous sources of external radiation. In hadronic models (e.g., Mücke et al. (2003)) the high-frequency peak is due to synchrotron emission of accelerated protons or due to photo-pion reactions, while the low-frequency peak is synchrotron emission of secondary electrons and positrons or, alternatively, of co-accelerated electrons. The hadronic models are usually applied to explaining TeV blazars, because making them reasonably efficient in $\mathrm{GeV}$ range is difficult. None of the listed models can readily explain both $\mathrm{GeV}$ and $\mathrm{TeV}$ blazars, so that the two populations may have different emission mechanisms.

Another example of relativistic outflows, Gamma-Ray Bursts (see, e.g., Zhang (2018) for a review), are always observed as point sources, but there is little doubt that at the late (so-called afterglow) stage their emission comes from highly relativistic shock, which gradually decelerates propagating into the circumburst material and produces radiation via the SSC mechanism. Presence of two components (lower-energy synchrotron and higher-energy self-Compton) was predicted long ago (Derishev et al., 2001; Sari \& Esin, 2001; Zhang \& Mészáros, 2001), but the observational confirmation came only recently (MAGIC Collaboration et al., 2019; Abdalla et al., 2019). Although broad-band GRB spectra do not demonstrate two humps as clearly as blazar spectra do, the physical setup for their radiation mechanisms is similar and therefore some conclusions derived from analysis of these observations may be transferred from GRB field to blazar field.

\section{Typical blazar parameters in spectral modeling}

In the following analysis, we will use typical blazar parameters summarized in Table (1). Parameters of specific sources may deviate from values in the table by some factor, but the conclusions do not change qualitatively. A special kind of such difference is extreme ultra-fast variability with timescales up to minutes, that was observed in few cases Albert et al. (2007); Aharonian et al. (2007). This phenomenon may be unrelated to regular variability and is not discussed here.

In this paper we use notation $\varepsilon_{I C}$ for the position of high-frequency peak and use the term Compton dominance for the ratio of power in the high-frequency peak to power in the low-frequency peak. This naming convention merely reflects the fact that in the vast majority of models the highfrequency emission is considered to be of inverse Compton origin, but we do not require this and consider other options on equal grounds.

Although models may differ in explaining the high-frequency peak, they all attribute the lowfrequency peak to synchrotron emission of electrons. In the case of synchrotron radiation one relates its frequency to the the Lorentz factor of radiating particles and the magnetic field strength,

$$
B=\frac{1}{\Gamma \gamma^{2}} \frac{m c}{\hbar e} \varepsilon_{s y} .
$$

Here $\gamma$ and $m$ are the Lorentz factor and mass of the radiating particles - we keep in mind that not only electrons and positrons, but also protons and muons may emit synchrotron radiation.

Using the synchrotron cooling rate (in the fluid-comoving frame)

$$
\dot{E}=-\frac{4}{9}\left(\frac{e^{2}}{m c^{2}}\right)^{2} \gamma^{2} B^{2} c
$$




\begin{tabular}{lll} 
Quantity & GeV blazars & TeV blazars \\
\hline Known from observations: & $\Gamma \sim 10$ & $\Gamma \sim 10$ \\
jets' Lorentz factors & $\Delta t \sim 1 \mathrm{day}$ & $\Delta t \sim 1 \mathrm{hour}$ \\
$\quad$ variability timescale & $L \sim 3 \times 10^{47} \mathrm{erg} / \mathrm{s}$ & $L \sim 10^{45} \mathrm{erg} / \mathrm{s}$ \\
apparent (isotropic equivalent) luminosity & $\varepsilon_{s y} \sim 1 \mathrm{eV}$ & $\varepsilon_{s y} \sim 1 \mathrm{keV}$ \\
position of low-frequency peak in SED & $\varepsilon_{I C} \sim 1 \mathrm{GeV}$ & $\varepsilon_{I C} \sim 1 \mathrm{TeV}$ \\
position of high-frequency peak in SED & $\eta_{I C} \sim 30$ & $\eta_{I C} \sim 0.3$ \\
Compton dominance & & \\
Derived: & $\sim 3 \times 10^{17} \mathrm{~cm}$ & $\sim 10^{16} \mathrm{~cm}$ \\
lab-frame size of emitting region, $R \sim \Gamma^{2} c \Delta t$ & $\sim 0.3 \mathrm{erg} / \mathrm{cm}^{3}$ \\
radiation energy density, $w_{\text {rad }} \sim L /\left(4 \pi \Gamma^{2} R^{2} c\right)$ & $\sim 0.1 \mathrm{erg} / \mathrm{cm}^{3}$ & \\
magnetic field in equipartition with radiation, & & \\
$B_{e q}^{2} /(8 \pi)=w_{\text {rad }}$ & $B_{e q} \sim 1.5 \mathrm{G}$ & $B_{e q} \sim 3 \mathrm{G}$
\end{tabular}

Table 1: Parameters for typical representatives of $\mathrm{GeV}$ and $\mathrm{TeV}$ blazar populations. Note that in the fluidcomoving frame the variability timescale is $\sim \Gamma \Delta t$ and the size of emitting region is $\sim R / \Gamma \sim \Gamma c \Delta t$.

and Eq. (2.1) one expresses the fast cooling condition $|\dot{E}| \Gamma \Delta t>\gamma m c^{2}$ in terms of the synchrotron photon energy and the magnetic field strength (Aharonian, 2000):

$$
\Delta t>\frac{9 m c}{4}\left(\frac{m c^{2}}{e^{2}}\right)^{2}\left(\frac{1}{\Gamma \varepsilon_{s y}} \frac{\hbar e}{m c}\right)^{1 / 2} B^{-3 / 2} \simeq 1 \text { day } \times \Gamma^{-1 / 2}\left(\frac{m}{m_{e}}\right)^{5 / 2}\left(\frac{\varepsilon_{s y}}{1 \mathrm{eV}}\right)^{-1 / 2}\left(\frac{B}{1 \mathrm{G}}\right)^{-3 / 2} .
$$

We note that electrons in blazars are in the fast cooling regime (maybe marginally in some cases).

Both synchrotron and inverse Compton radiation are due to interaction of particles with electromagnetic field, where the same cross-section applies in the classical limit. Therefore, the ratio of IC to synchrotron luminosities (or Compton dominance) can be expressed via the ratio of energy densities in the seed radiation (synchrotron in case of SSC) and in the magnetic field:

$$
\eta_{I C} \simeq \kappa_{K N}\left(w_{r a d} / w_{B}\right)
$$

Here $\kappa_{K N} \leq 1$ is the Klein-Nishina correction factor, which equals unity in Thomson regime.

Within the framework of the SSC model, TeV blazars produce inverse Compton peak in the Klein-Nishina regime, so that the energy of IC photons approximately equals to the energy of comptonizing electrons, i.e.,

$$
\gamma_{e}=\frac{\varepsilon_{I C}}{\Gamma m_{e} c^{2}} \sim 10^{5} \Rightarrow B \sim 1 \mathrm{G}
$$

and $w_{B} \sim 0.1 w_{\text {rad }}$. This leads to estimation of Compton dominance $\eta_{I C} \sim 10 \kappa_{K N}$ that is consistent with observations provided the Klein-Nishina correction factor is small enough. In contrast, $\mathrm{GeV}$ blazars produce inverse Compton peak in the Thomson regime and each upscattering results in $\gamma_{e}^{2}$ times increase in photon's energy, so that

$$
\gamma_{e}=\sqrt{\varepsilon_{I C} / \varepsilon_{s y}} \sim 3 \times 10^{4} \Rightarrow B \sim 10^{-3} \mathrm{G}
$$


With this estimate of the magnetic field strength $w_{B} \sim 10^{-6} w_{\text {rad }}$, resulting in enormously large and certainly unrealistic Compton dominance. So far there is no solution to this discrepancy.

In the ERC model, GeV blazars also produce the high-frequency peak in the Thomson regime, that gives

$$
\gamma_{e}=\frac{1}{\Gamma} \sqrt{\varepsilon_{I C} / \varepsilon_{\text {disk,BLR }}} \sim 3 \times 10^{2} \Rightarrow B \sim 10 G
$$

assuming $\sim 100 \mathrm{eV}$ external photons. This estimate results in $w_{B} \sim 30 w_{\text {rad }}$, so that the jet's Poynting flux is much larger than the radiation energy flux and the overall radiative efficiency cannot be high.

The hadronic models, in general, suffer from low efficiency. Substituting $m$ with the proton mass $m_{p}$ in Eq. (2.3), we find that fast cooling regime for proton synchrotron emission in both $\mathrm{GeV}$ and TeV blazars requires $B \sim 300 \mathrm{G}$. With such a strong magnetic field the jets would be entirely Poynting-flux dominated, with radiation contributing only $w_{\text {rad }} / w_{B} \sim 10^{-4}$ fraction to the total power. With the optimal choice $B \sim 20 \mathrm{G}$ one achieves (in the slow-cooling regime) the largest possible efficiency $\sim 0.5$ percent for the proton-synchrotron hadronic models of blazars ${ }^{1}$.

Choosing in favor of photo-pion reactions or Bethe-Heitler pair production as the means to extract energy from accelerated protons, one builds up several factors contributing to overall inefficiency: (i) the source must be transparent to gamma-ray radiation, but the cross-section for protonphoton interactions is one-two orders of magnitude smaller than two-photon pair-production crosssection, (ii) only a small fraction $\left(\sim 0.1\right.$ for photo-pion reactions and $\sim 10^{-3}$ for Bethe-Heitler process) of proton energy can be converted to electromagnetic radiation in each proton-photon interaction, (iii) proton synchrotron radiation is always a competitor in draining protons' energy. Small efficiency is unavoidable even if all these factors arrange at their optimal values, and there is no reason why they should.

\section{Acceleration rate issue}

One further step in understanding emission from relativistic outflows is gaining insight into the acceleration process that produces the kind of distribution of energetic particles one needs to inject into the emitting zone in order to explain the observed spectra.

Usual depiction of a non-thermal particle distribution describes it as consisting of two parts. The majority of particles constitutes "thermal-like" core of the distribution and the most energetic of them enter acceleration process and form a power-law tail with high-energy cut-off (at the cutoff energy either acceleration becomes inefficient or the particles simply do not have enough time to gain more energy).

If one assigns the low-frequency peak to the synchrotron radiation of "thermal-like" particles, then modeling of SED above the peak requires rather soft injection function with power-law index $p \simeq-(2.5 \div 4)$ and one or two breaks (e.g., Hayashida et al. (2015), Abdo et al. (2011)). This is at odds even with diffusive shock acceleration theories ${ }^{2}$, which predict single power-law with

\footnotetext{
${ }^{1}$ Modeling of ultra-fast blazar flares implies - out of necessity - very small size of emitting zone and thus allows to achieve higher efficiency of proton synchrotron radiation (Khangulyan et al., 2013).

${ }^{2}$ Some acceleration theories, for example magnetic reconnection (Werner et al., 2016) and converter mechanism (Derishev et al., 2003), predict non-integrable power-law tail with $p>-2$. In these cases the synchrotron peak must be assigned to the cut-off in the power-law tail rather than to the "thermal-like" particles.
} 
$p \simeq-2.2$ for relativistic shocks (e.g., Achterberg et al. (2001)) and $p \simeq-2$ for non-relativistic ones (e.g., Bell (1978)), whereas these predictions pass observational tests in the Crab nebula (e.g., Meyer et al. (2010)) and in supernova remnants (e.g., Zirakashvili \& Aharonian (2010)). One also has to explain why in $\mathrm{GeV}$ and $\mathrm{TeV}$ blazars the energy of "thermal-like" particles is greatly different despite very similar bulk flow parameters (the Lorentz factor and the magnetic field strength). So far there is no idea how to overcome these issues.

Another possibility is that the synchrotron peak is due to particles which pile up at the energy where radiative losses balance acceleration. Let's explore what is the smallest synchrotron frequency still consistent with radiation-limited acceleration (see Derishev (2007) for details). We parametrize acceleration rate as $\dot{E}=\eta e B c$ and balance it with losses (Eq. 2.2) to obtain the maximum particle's Lorentz factor and then the corresponding energy of synchrotron photons

$$
\gamma_{\max }^{2} \simeq \frac{9}{4}\left(\frac{m c^{2}}{e^{2}}\right)^{2} \frac{\eta e}{B} \quad \Rightarrow \quad \varepsilon_{s y, \max } \simeq \frac{9}{4} \Gamma \eta \frac{m c^{2}}{\alpha}
$$

where $\alpha \simeq 1 / 137$ is the fine structure constant. For diffusive shock acceleration with Bohm diffusion coefficient $\eta \simeq\left(v_{s h} / c\right)^{2}$ (Lagage \& Cesarsky, 1983), that naively results in $\eta \simeq 1$ for relativistic shocks.

Slower acceleration is achieved by increasing particles' scattering length. If the magnetic field changes its direction on scales $\sim \lambda_{B}$, much smaller than the gyroradius $r_{g}$, then acceleration efficiency can be estimated as $\eta \sim\left(\lambda_{B} / r_{g}\right) \ll 1$. Thus, decreasing magnetic field correlation length will shift the synchrotron peak to smaller frequencies, but only until $\lambda_{B} \gtrsim r_{g} / \gamma_{e}$. At still smaller values of $\lambda_{B}$ one encounters transition to undulator regime and the characteristic frequency of radiation starts to increase, $\omega \propto \lambda_{B}^{-1}$, so that assumption $\eta=1 / \gamma_{e}$ results in the minimum possible (within this framework) energy of photons at the synchrotron peak. Therefore,

$$
\varepsilon_{s y} \gtrsim \Gamma \frac{m_{e} c^{2}}{\alpha \gamma_{e}} .
$$

Assuming that blazars are as bad accelerators as possible, one can barely explain the position of low-frequency peak at $\sim 1 \mathrm{keV}$ in $\mathrm{TeV}$ blazars. At the same time, there is no way to reach eV energy range required to explain $\mathrm{GeV}$ blazars, even if corrections for the cooling due to IC emission are taken into account.

Of course, the inconsistencies listed above do not apply to the case where the particles emitting synchrotron radiation at the low-frequency peak are secondaries. For example, in hadronic models secondary electrons and positrons are produced in some kind of cascading process, not directly related to acceleration, from high-energy primaries responsible for emission at the high-frequency peak. Therefore, the hadronic models avoid this trap, but they are bound to have low efficiency.

Keeping in mind the difficulties of various kinds in existing blazar models, we note a nice coincidence between the high-frequency peak positions in $\mathrm{GeV}$ and $\mathrm{TeV}$ blazars on one hand and, on the other hand, the maximum synchrotron photon energy for electrons and protons accelerated at the largest possible rate $\eta \simeq 1$ (Eq. (3.1) gives then $\sim 1 \mathrm{GeV}$ and $\sim 2 \mathrm{TeV}$, respectively). It is therefore tempting to assume that it is the high-frequency peak in the spectra of both $\mathrm{GeV}$ and $\mathrm{TeV}$ blazars that should be attributed to synchrotron emission of primary particles, with the radiating 
particles being electrons and protons, respectively. This assumption places blazars among the extremely efficient accelerators, exactly as one would expect for the objects, where internal motions occur at nearly the speed of light. In the regime of radiation-limited acceleration, the peak synchrotron frequency does not depend on the magnetic field strength and the equipartition field will suffice, solving another possible issue with the required magnetic field energy density deviating too much from its natural value and thus reducing efficiency.

In this picture the low-frequency peak should be produced by secondary particles and it is worth examining whether this violates any restrictions imposed by blazars' parameters. In any case, such a setup implies strong back reaction from high-energy radiation via production of large number of secondary particles. In the next section we discuss how recent observations of sub-TeV emission from GRBs shed light on radiative back reaction in relativistic outflows.

\section{A clue from Gamma-Ray Bursts}

In an attempt to consider radiation processes, particle acceleration and their interplay, the pair-balance model for relativistic shocks was suggested (see Derishev \& Piran (2016) for details). The key component of this model is acceleration - radiation feedback, which can be described as an infinite loop incorporating the following sequence of events (see Fig. 2). We start with highenergy photons produced in the downstream emitting zone (they are seen in the broad-band spectra and for this moment we do not specify their origin). Some of them are absorbed in the upstream via two-photon pair production when they interact with abundant low-frequency photons escaping from the emitting zone (say, synchrotron radiation from the same population of electrons). Being captured by the upstream's magnetic field, the secondary pairs are Lorentz-boosted to much higher energy (as viewed from the downstream comoving frame). The boosted pairs are carried to the downstream, where they emit both synchrotron and IC radiation. In response to the increased supply of more energetic particles into the emitting zone, the IC peak goes up both in power and in photon energy. With a larger number of more energetic photons being produced, more photons are absorbed in the upstream. Secondary pairs transfer their momentum to the flow and accelerate upstream fluid before the shock, reducing (or eliminating) velocity jump. Acceleration of the upstream fluid in the lab frame means its deceleration in the downstream frame, so the Lorentz boost for secondary pairs becomes smaller. In response, the IC peak goes down both in power and in photon energy, so that fewer IC photons are absorbed in the upstream. Then the loop repeats until a steady state is reached.

In this model with radiation feedback, the result of shock self-balancing is that the typical energy of radiating electrons (the one that corresponds to the electrons contributing most to the energy density) settles at a value, which places production of IC peak photons to the border between Klein-Nishina and Thomson regimes, barely above the threshold for two-photon pair production when the IC photons interact with the synchrotron ones. Also, a significant fraction of IC radiation is absorbed in the upstream and modifies the shock structure, creating a smooth velocity profile instead of velocity jump. For this type of shock structure there is no room for diffusive shock acceleration as the velocity difference across the electrons' mean free path is very small compared to the shock speed. Instead, acceleration and radiation are the two sides of one and the same cooperative process. 


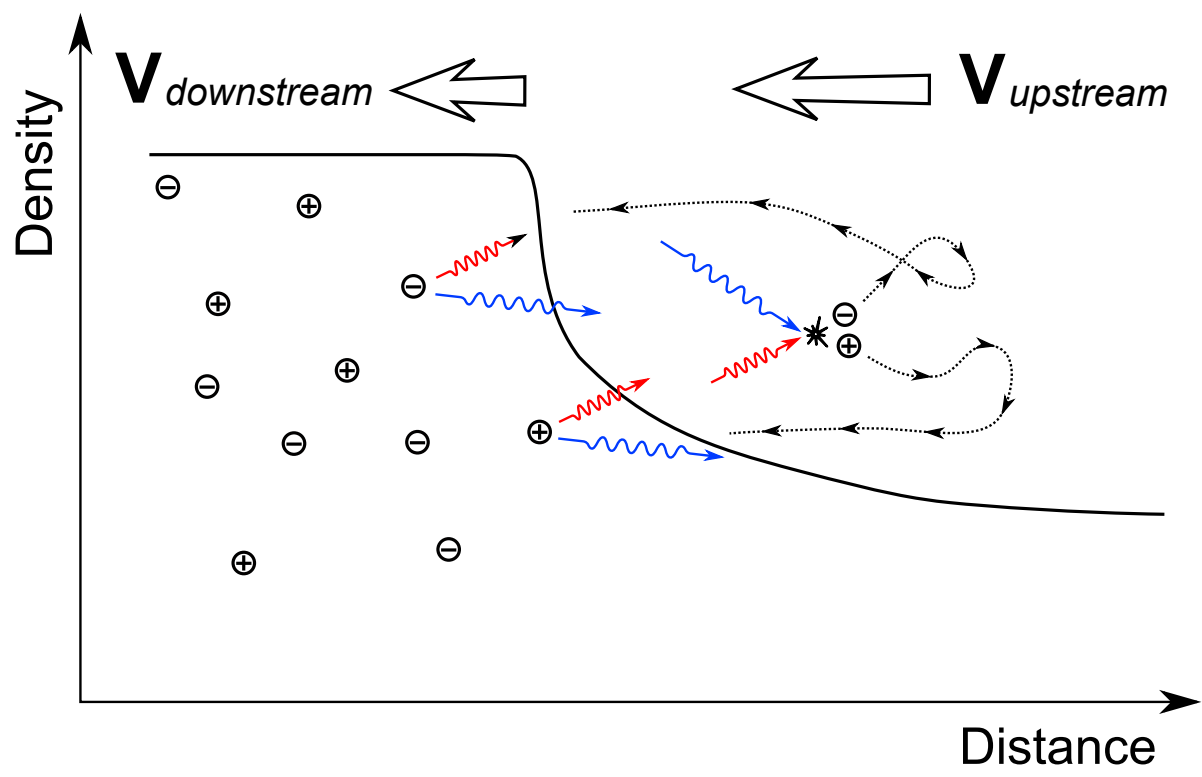

Figure 2: Processes involved into pair-balance model, as seen in the shock frame. Electrons ("-") and positrons ("+") in the downstream produce synchrotron (short red arrows) and inverse Compton (long blue arrows) photons. Some of the IC photons annihilate with synchrotron photons in the upstream (asterisk), producing electron-positron pairs. Magnetic field in the upstream captures the pairs; upstream fluid takes their momentum, decelerates and transports the pairs to the downstream.

Recently, observations of GRBs in sub-TeV range (MAGIC Collaboration et al., 2019; Abdalla et al., 2019) provided support for the pair-balance model as follows from analysis of preliminary data (Derishev \& Piran, 2019). Here we give a brief summary of these results.

At the time of observation ( $\sim 100$ seconds from the trigger), the shock Lorentz factor is limited both from below and from above: $\Gamma \gtrsim 100$ is required to avoid strong two-photon absorption inside the emitting zone and $\Gamma \lesssim 150$ follows from shock deceleration law assuming reasonable kinetic energy of the ejecta, $\lesssim 10^{54} \mathrm{erg}$ isotropic equivalent.

Within the general SSC framework, without a priory knowledge about the actual regime of comptonization, one may take two estimates from Eqs. (2.5), (2.6) and choose the smaller of them; the choice will tell the regime of comptonization. For the parameters of GRB 190114C (the synchrotron peak at $\sim 10 \mathrm{keV}$ and the IC peak at $\sim 300 \mathrm{GeV}$ ) both expressions give approximately the same value for the Lorentz factor of emitting electrons, $\gamma_{e} \simeq 1.5 \times 10^{4}$, i.e., the sub-TeV radiation was produced on the border between $\mathrm{KN}$ and Thomson regimes.

Given this fact and the observed Compton dominance $\eta_{I C} \sim 1 / 3$, we find from Eq. (2.4) that the magnetic field energy must be of the order of synchrotron radiation energy density and, as long as electrons are in the fast cooling regime, this requires that the fractions of shock energy going into particle acceleration and into the magnetic field generation are of the same order, $\varepsilon_{e} \sim \varepsilon_{B}$. Taken together with the results of numerical simulations (e.g., Sironi \& Spitkovsky (2011)), that typically produce $\varepsilon_{B} \sim 10^{-2}$, this conclusion means that $\varepsilon_{e}$ and hence the overall radiative efficiency at the early afterglow phase are of the order of $10^{-2}$, implying very large shock kinetic energy well in excess of $10^{54} \mathrm{erg}$ (isotropic equivalent). However, one can relax this requirement assuming that 
there is moderate two-photon absorption of IC radiation inside the emitting zone, that corresponds to a larger intrinsic Compton dominance and therefore to a larger $\varepsilon_{e} / \varepsilon_{B}$ ratio.

The above conclusions - that radiation at the IC peak is produced on the border between KN and Thomson regimes and that a large fraction of high-energy IC radiation is absorbed upstream of the shock - are exactly the two key predictions of the pair-balance model. Unless it is simply a coincidence, we finally catch a glimpse of the micro-physical machinery that relates radiation and acceleration processes in relativistic flows and there is no immediate obstacle for a similar machinery to work in blazars as well.

\section{Discussion}

In this paper we discuss several models, which were put forward to explain broad-band emission from relativistic outflows (most prominently from blazars and GRBs) under certain model assumptions about the injection function for the radiating particles. All for them have limited success, explaining a part of source population but not the whole population. On the other hand, all of the models face serious difficulties when one tries to reconcile model assumptions about particle injection with predictions of particle acceleration models. Therefore, no full solution exists for the problem of calculating spectra of non-thermal radiation from relativistic outflows.

The parameters of GRB emitting zone extracted from recent observations of sub-TeV emission favor the pair-balance model of radiatively efficient relativistic shocks. One of the main elements of this model is the back reaction of high-energy radiation produced by non-thermal particles onto shock structure and then onto particle acceleration. Based on the support received by the pairbalance model and judging from physical similarity between blazars and GRBs as well as from apparent inconsistency between blazar emission models and particle acceleration models, we conclude that in extreme environments the ties between particle acceleration and radiation processes are so close, that they cannot be considered separately. Construction of models, which consistently take into account radiation processes, particle acceleration processes and their interplay, is a challenging task and may be called a new, emerging, branch of high-energy astrophysics.

\section{Acknowledgement}

This research was supported by the Russian Science Foundation grant No 16-12-10528. I thank the referee for useful comments.

\section{References}

Abdalla H., et al., 2019, Nature, 575, 464

Abdo A. A., et al., 2010, ApJ, 716, 30

Abdo A. A., et al., 2011, ApJ, 736, 131

Achterberg A., Gallant Y. A., Kirk J. G., Guthmann A. W., 2001, MNRAS, 328, 393

Aharonian F. A., 2000, New Astronomy, 5, 377 
Aharonian F., et al., 2007, ApJL, 664, L71

Albert J., et al., 2007, ApJ, 669, 862

Bell A. R., 1978, MNRAS, 182, 147

Derishev E. V., 2007, Ap\&SS, 309, 157

Derishev E. V., Piran T., 2016, MNRAS, 460, 2036

Derishev E., Piran T., 2019, ApJL, 880, L27

Derishev E. V., Kocharovsky V. V., Kocharovsky V. V., 2001, Astronomy and Astrophysics, 372, 1071

Derishev E. V., Aharonian F. A., Kocharovsky V. V., Kocharovsky V. V., 2003, Phys Rev D, 68, 043003

Finke J. D., 2013, The Astrophysical Journal, 763, 134

Ghisellini G., Maraschi L., 1989, ApJ, 340, 181

Hayashida M., et al., 2015, ApJ, 807, 79

Khangulyan D. V., Barkov M. V., Bosch-Ramon V., Aharonian F. A., Dorodnitsyn A. V., 2013, ApJ, 774, 113

Lagage P. O., Cesarsky C. J., 1983, Astronomy and Astrophysics, 125, 249

MAGIC Collaboration et al., 2019, Nature, 575, 455

Meyer M., Horns D., Zechlin H. S., 2010, Astronomy and Astrophysics, 523, A2

Mücke A., Protheroe R. J., Engel R., Rachen J. P., Stanev T., 2003, Astroparticle Physics, 18, 593

Sari R., Esin A. A., 2001, ApJ, 548, 787

Sikora M., Begelman M. C., Rees M. J., 1994, ApJ, 421, 153

Sironi L., Spitkovsky A., 2011, ApJ, 726, 75

Urry C. M., Padovani P., 1995, Publications of the Astronomical Society of the Pacific, 107, 803

Werner G. R., Uzdensky D. A., Cerutti B., Nalewajko K., Begelman M. C., 2016, ApJL, 816, L8

Zhang B., 2018, The Physics of Gamma-Ray Bursts, doi:10.1017/9781139226530.

Zhang B., Mészáros P., 2001, ApJ, 559, 110

Zirakashvili V. N., Aharonian F. A., 2010, ApJ, 708, 965 